\title{
Renewable energies projects selection: block criteria systematization with AHP and Entropy- MOORA methods in MCDM
}

\author{
Iliyan Petrov, ${ }^{1}$ \\ ${ }^{1}$ Bulgarian Academy of Sciences, Institute of Information and Communication \\ Technologies, Dep. "Information Processes and Decision Support Systems", akad. Georgi \\ Bonchev srt., bl. 2, 1113 Sofia, Bulgaria
}

\begin{abstract}
EU countries face two major challenges in the energy sector the control of carbon emissions and the high dependence on imported primary energy sources. Both European Commission and national governments actively develop, update and upgrade strategies and plans for the energy sector. They aim to ensure the gradual reaching of consecutive targets for increasing the weight of Renewable Energies (RE) in the European primary energy mix. The selection of Renewable Energy investment projects in these sectors involves different groups of experts who have to consider the specifics of different countries, regions, and technologies. The Decision-making process becomes more complicated, as in addition to political goals it has to ensure the efficiency and competitiveness of European economies. Several conflicting objectives have to be balanced with complex social, economic, technological, and environmental factors. Our study explores the application of combined methods and evaluation techniques for improving the assessment and selection of prospective RE projects. We implement an innovative integration of the Analytical Hierarchy Process (AHP) for block criteria systematization combined with objective Shannon Entropy in the MOORA evaluation.
\end{abstract}

\section{Introduction}

Multi-Criteria Decision Making (MCDM) is one of the main disciplines in operations analysis which employs different subjective (expert-dependant) and objective (data-driven) methods for assessing the quality and performance of complex systems in many areas. On one hand, the risks of relying on experts' opinions are determined by the subjectivity of human experiences and preferences, while, on the other hand, the data-driven selections depend on the quality of data and practically neglect the users' needs. One-sided subjective or analytical methods may lead to uncertainty in the evaluation decision-making process.

\footnotetext{
* Corresponding author: petrovindex@gmail.com
} 
The Multi-Objective Optimization with Ratio Analysis (MOORA) is a popular approach with a compensatory concept for handling complex tasks for the assessment of numerous alternatives with multiple controversial criteria [1-6]. It is more transparent, intuitive, and easy for computation than other concepts, such as TOPSIS [7] and VIKOR [8], and allows to combine it easily with other methods without over-engineering the calculations.

RE energy projects can be based on different technologies and management systems and their functioning can be assessed with different criteria and methods [9-13].

\section{Objective definition of criteria weights with Entropy-MOORA}

A combination of MOORA with the Information Entropy concept allows performing an objective definition of weights for evaluation criteria [14-16]. It is used for different MCDM problems and includes several consecutive steps. In this paper, we use the essentials of common MCDM methodology aspects developed in our previous work [24].

Step 1. This step prepares the data for the selection process in the following sub-steps:

Step 1.1. Definition of the Basic Decision Matrix (BDM) comprising a list of " $m$ " target alternatives $\left(A_{i}\right)$ which are evaluated with a set of " $n$ " independent criteria $\left(C_{j}\right)$ :

$$
B D M_{m \times n}=\left[x_{i j}\right]_{m \times n} \text {, where } i=1,2, \ldots, m ; j=1,2, \ldots, n
$$

In this study, we use an existing data-set [17] containing 13 alternatives RE projects and 7 selection attributes shown in Table 1 .

Table 1. Target alternatives and evaluation criteria for selection of RE projects

\begin{tabular}{|c|c|c|c|c|c|}
\hline \multicolumn{2}{|r|}{ Alternative RE projects } & \multicolumn{3}{|r|}{ Criteria } & \multirow{2}{*}{\begin{tabular}{|l|} 
Unit \\
MW
\end{tabular}} \\
\hline WA1 & Wind power $\mathrm{P} \leq 5 \mathrm{MW}$ & $\mathrm{C} 1$ & $\mathrm{P}$ & Power & \\
\hline WA2 & Wind power $5 \leq \mathrm{P} \leq 10 \mathrm{MW}$ & $\mathrm{C} 2$ & $\mathrm{OH}$ & Operating Hours/year & $1000 \mathrm{~h} / \mathrm{y}$ \\
\hline WA3 & Wind power $10 \leq \mathrm{P} \leq 50 \mathrm{MW}$ & $\mathrm{C} 3$ & UP & Utilisation Period & years \\
\hline HA4 & Hydroelectric $\mathrm{P} \leq 10 \leq \mathrm{MW}$ & $\mathrm{C} 4$ & $\mathrm{tCO} 2 / \mathrm{y}$ & Avoided CO2 emissions & $\mathrm{Mt} / \mathrm{y}$ \\
\hline HA5 & Hydroelectric $10 \leq \mathrm{P} \leq 25 \mathrm{MW}$ & $\mathrm{C} 5$ & IR & Investment Ratio & $€ / K W$ \\
\hline HA6 & Hydroelectric $25 \leq \mathrm{P} \leq 50 \mathrm{MW}$ & C6 & $\mathrm{CP}$ & Construction period & years \\
\hline SA7 & Solar Thermo-electric P $10 \geq \mathrm{MW}$ & $\mathrm{C} 7$ & $\mathrm{O} \& \mathrm{MC}$ & Oper. \& Mainten. Cost & $€ / M W h$ \\
\hline BA8 & Biomass (energetic cultivations) $\leq 5 \mathrm{MW}$ & & & & \\
\hline BA9 & Biomass (forest $\&$ agro wastes) $\mathrm{P} \leq 5 \mathrm{MW}$ & & & & \\
\hline BA10 & Biomass (farm industry wastes) $\mathrm{P} \leq 5 \mathrm{MW}$ & & & & \\
\hline BA11 & Biomass (forest industry wastes) $\mathrm{P} \leq 5 \mathrm{MW}$ & & & & \\
\hline BA12 & $\begin{array}{l}\text { Biomass (co-comb.in conv. central) } \mathrm{P} \geq 50 \\
\text { MW }\end{array}$ & & & & \\
\hline
\end{tabular}

The number of alternatives " $m$ " is not limited, but it is preferable to perform a preliminary selection according to the preferences and restraints of DM. This data-set contains projects of different scales based on mature and widely accessible technologies based on four types of RE for producing electricity: wind power (W), hydropower $(\mathrm{H})$, solar (S), and biomass (B). Table 2 contains the Normalized Decision Matrix (NDM)

Step 1.2. Calculating the proportions of relative "basic weights $p$ " for each value of $x_{i j}$ in $\mathrm{C}(i)$ and formation of Normalized Decision Matrix $(N D M)$ with $\sum_{i=1}^{m} p_{i j}=1$ :

$$
\begin{aligned}
& p_{i j}=x_{i j} / \sum_{i=1}^{m} p_{i j} \\
& N D M=\left[p_{i j}\right]_{m \times n}=\left[\begin{array}{ll}
p_{11} & p_{1 n} \\
p_{m 1} & p_{m n}
\end{array}\right], \text { where } i=1,2, \ldots, m ; j=1,2, \ldots, n .
\end{aligned}
$$

Step 1.2. Calculation of the total nominal Shannon Entropy [18] for each criterion vectorcolumn " $j$ " as a sum of the " $i$ " individual entropies for the " $m$ " target alternatives:

$$
\text { SEnom }_{j}=-\sum_{i=1}^{m} s e_{j}=-\sum_{i=1}^{m} p_{i j} \cdot \log _{2}\left(p_{p j}\right)
$$

Step 1.3. Calculation of maximal Shannon Entropy value for a set of " $m$ " alternatives in which all elements have equal relative weights, i.e. $p_{i j}=1 / \mathrm{m}$ :

$$
\operatorname{SEmax}(m)=-\sum_{i}^{m}\left(1 / m \cdot \log _{2} 1 / m\right)=\log _{2} m
$$


Step 1.4. Normalization of nominal entropy $S E n o m_{j}$ by comparing it to $\operatorname{SEmax}(m)$ :

SEnorm $_{j}=e_{j}=\operatorname{SEnom}_{j} / \operatorname{SEmax}(m)=-\left(\log _{2} m\right)^{-1} \sum_{i=1}^{m} p_{i j} \cdot \log _{2}\left(p_{i j}\right)$

Step 1.5. Calculation of the sum of the normalized criteria entropies $e_{j}=S E n o r m_{j}$ and defining their real entropy criteria weights $\left(r e c w_{j}\right)$ for each criterion $C_{j}$ :

$$
\left.\operatorname{recw}_{j}=e_{j} / \sum_{i=1}^{n}=e_{j}\right)
$$

Table 2. Basic Decision Matrix (BDM)

\begin{tabular}{|c|ccccccc|}
\hline Criteria (n) & C1 & C2 & C3 & C4 & C5 & C6 & C7 \\
\hline & P & OH & UP & tCO2/y & IR & CP & O\&MC \\
\hline & MW & $1000 \mathrm{~h} / \mathrm{y}$ & years & Mtons/ & €/KW & years & E/MWh \\
Alternatives (m) & Benefit & Benefit & Benefit & Benefit & Cost & Cost & Cost \\
\hline WA1 & 5 & 2,35 & 20 & 1929936 & 937 & 1 & 1,470 \\
WA2 & 10 & 2,35 & 20 & 3213560 & 937 & 1 & 1,470 \\
WA3 & 25 & 2,35 & 20 & 9649680 & 937 & 1 & 1,510 \\
\hline HA4 & 5 & 3,10 & 25 & 472812 & 1500 & 1,5 & 1,450 \\
HA5 & 20 & 2.00 & 25 & 255490 & 700 & 2 & 0,700 \\
HA6 & 35 & 2,59 & 25 & 255490 & 601 & 2,5 & 0,600 \\
\hline SA7 & 50 & 75,00 & 25 & 482856 & 5000 & 2 & 4,200 \\
\hline BA8 & 5 & 75,00 & 15 & 2524643 & 1803 & 1 & 7,106 \\
BA9 & 5 & 75,00 & 15 & 2524643 & 1803 & 1 & 5,425 \\
BA10 & 5 & 75,00 & 15 & 2524643 & 1803 & 1 & 5,425 \\
BA11 & 5 & 7500 & 15 & 2524643 & 1803 & 1 & 2,813 \\
BA12 & 56 & 75,00 & 20 & 4839548 & 856 & 1 & 4,560 \\
BA13 & 2 & 7,00 & 20 & 5905270 & 1503 & 1,5 & 2,512 \\
\hline
\end{tabular}

Based on additional data treatment whose aim is to substitute the genuine results from the transformation of empiric data with the "idea" to enhance the difference between criteria through a "divergence parameter" $d_{j}$ which represents an arithmetic difference between the normalized maximal entropy $S E \max (m)$ expressed as "1" minus $S E n o r m_{j}$ expressed as $" e_{j}$ "as defined in eq. (6-7):

$$
\begin{aligned}
& d_{j}=1-e_{j} \\
& \operatorname{decw}_{j}=d_{j} / \sum_{i=1}^{m} d_{j}=\left(1-e_{j}\right) / \sum_{i=1}^{m}\left(1-e_{j}\right)
\end{aligned}
$$

The results of Steps 3.1-6 are displayed in Table 3.

Table 3. Real and differed entropy weights in stand-alone Entropy approach

\begin{tabular}{|c|c|c|c|c|ccc|}
\hline Criteria & $\mathbf{P}$ & $\mathbf{O H}$ & $\mathbf{U P}$ & tCO2/y & IR & CP & O\&MC \\
\hline WA1 & 0,121 & 0,038 & 0,285 & 0,22 & 0,206 & 0,236 & 0,178 \\
$\ldots$ & $\ldots$ & $\ldots$ & $\ldots$ & $\ldots$ & $\ldots$ & $\ldots$ & $\ldots$ \\
BA13 & 0,060 & 0,090 & 0,285 & 0,42 & 0,279 & 0,304 & 0,254 \\
\hline SEnom & 3,033 & 2,858 & 3,672 & 3,15 & 3,431 & 3,612 & 3,376 \\
SEmax & 3,700 & 3,700 & 3,700 & 3,700 & 3,700 & 3,700 & 3,700 \\
\hline SEnorm $=\mathrm{e}_{j}$ & 0,820 & 0,772 & 0,992 & 0,852 & 0,927 & 0,976 & 0,912 \\
recw $_{j}$ & 0,131 & 0,124 & 0,159 & 0,136 & 0,148 & 0,156 & 0,146 \\
\hline$d_{j}=1-e_{j}$ & 0,180 & 0,228 & 0,008 & 0,148 & 0,073 & 0,024 & 0,088 \\
decw $_{j}$ & 0,241 & 0,305 & 0,010 & 0,197 & 0,097 & 0,032 & 0,117 \\
\hline
\end{tabular}

The substitution of the genuine objective "real entropy criteria weights $\left(\operatorname{recw}_{j}\right)$ " with another "concentration-like set" of "differed entropy criteria weights $\left(\right.$ decw $\left._{j}\right)$ " produces very different results without taking into account the needs and preferences of users. If the discussion was purely theoretical, such extraction treatment might be regarded as an experiment. However, in any real situation, such artificially created reality dramatically transforms the positioning of all criteria distancing them from input empiric data. While the values of " $r e c w_{j}$ " are quite even, the values of " $d e c w_{j}$ " become very unequal. As a result, only 2 technical criteria ("Power" and "Operating hours") concentrate $54,6 \%$ of the total weights, while the "Utilization period" (1\%) and the "Construction period" $(3,2 \%)$ are practically neglected. In principle, in this treatment, the losers in a diversity context are 
transformed into winners in a concentration context. Such perturbation in many cases may be regarded as "data treatment", but in our opinion, the concept of "information extraction" from relative weights is illusive. If there are doubts about the capacity of the entropy approach a logical way-around would be to replace it with an approach that measures directly the opposite of diversity. In other words, to measure the concentration of information in each criterion rather than trying indirectly to "extract data" from entropy for judging about concentration. Actually, both approaches are purely technical and they may look nontransparent and technocratic. In fact, the most important task in MCDM is to focus on those parameters or functions which objectively correspond to the needs and preferences of users, as shown below in Section 3.

Step 2. Calculation of "normalized weighted values " $v$ " with real weights $\left(r_{i j} \cdot r e c w_{j}=\right.$ $\left.r v_{i j}\right)$ and with differed weighs $\left(r_{i j} \cdot d e c w_{j}=d v_{i j}\right)$ for constructing the respective Normalized Weighted Matrices $N W M$ with real and differed entropy criteria weights:

$$
\begin{aligned}
& N R W M_{m \times n}=\left[r_{i j} \cdot r e c w_{j}\right]_{m \times n}=\left[r v_{i j}\right]_{m \times n} \\
& N D W M_{m \times n}=\left[r_{i j} . \text { dec }_{j}\right]_{m \times n}=\left[d v_{i j}\right]_{m \times n}
\end{aligned}
$$

Step 3. Classification of criteria in "benefit (profit)" and "non-benefit (cost)" depending on how their attribute or save resources and performance. The optimization for "benefit (profit)" is expressed in maximal values and for "non-benefit(cost)" - in minimal values. Criteria C1-C4 are "benefit (profit)" type, while C5-C7 are "non-benefit (cost)" type.

Step 4. Calculation of MOORA Alternatives Assessments $\left(A A_{j}\right)$ for each alternative. In our case, we perform a comparison between the sets of $r e c w_{j}$ and $d c e w_{j}$ :

$$
\begin{aligned}
& \text { AArecw }_{j}=\sum_{j=1}^{g} r v_{i j}-\sum_{j=g+1}^{n} r v_{i j} \\
& \text { AAdecw }_{j}=\sum_{j=1}^{g} d v_{i j}-\sum_{j=g+1}^{n} d v_{i j}
\end{aligned}
$$

Step 5. Ranking the assessment results $M A A_{j}$ in descending order.

For better visualization, the results of Steps 5-8 for calculations in the traditional "SEMOORA" approach are displayed in Table 8, Section 4, here below.

\section{Optimizing AHP by systemizing the evaluation criteria in blocks}

The stand-alone Entropy approach described in Section 2 is data-driven and, in principle, excludes the participation of experts in defining the weights of evaluation criteria. However, a professional expertize is needed in many aspects - defining needs, pre-selecting target alternatives, selecting criteria, and applying evaluation methods. This report has not the possibility to explore the variety of topics related to expert activities, which are well discussed in numerous publications [19-22]. Our studies are focused on improving the traditional "Entropy \& MOORA" approaches by decomposing the initial set of criteria into smaller blocks for optimizing the assessment procedures and finding solutions that allow reflecting the users' needs and preferences.

In the algorithm of Section 2, an additional Step 1.0 is integrated for defining the weights of a limited number of blocks " $b$ " ( $b>1$ and, in our opinion, preferably $b \leq 5$ ) with the Analytical Hierarchy Process (AHP) method [23-24]. Such systematization allows to organize the expression of experts' opinions and to frame the subjectivity of individual judgments up to the stage of defining the importance of blocks. In case discussed in this paper, all criteria are grouped in 3 blocks:

1) "Technical - Block 1" - for all technical parameters $\left(\boldsymbol{C}_{\mathbf{1}}, \boldsymbol{C}_{\mathbf{2}}, \boldsymbol{C}_{\mathbf{3}}\right)$;

2) "Ecology - Bock 2" - for ecology and pollution control parameters $\left(\boldsymbol{C}_{\mathbf{4}}\right)$;

3) "Costs - Block 3"- for financial and time parameters related to the realisation of the project $\left(\boldsymbol{C}_{\mathbf{5}}, \boldsymbol{C}_{\mathbf{6}}, \boldsymbol{C}_{\mathbf{7}}\right)$. 
The logical systematization of criteria in blocks(groups) reduces the number of "pairscomparisons" and facilitates the hierarchical ranging of priorities, allowing to express with less confusion the individual preferences of DM. To define block weights a quadratic Hierarchy Preference Matrix $\left.\operatorname{HPM}_{(b} x_{b} b\right)$ is constructed in which the values reflect the judgments of DM according to the traditional AHP scale. The values of "1-3-5-7-9" correspond to pivot verbal judgments "equal importance - moderate importance - strong importance - very strong importance - top importance”. Interim values (2-4-6-8) can be used for more precision. The comparison of a single block $\left(b_{e}\right)$ with itself results in " 1 ", so the main diagonal's cells in $\operatorname{HPM}_{(b x b)}$ are fixed to " 1 ". If the hierarchy preference " $h p$ " of "Technical v/s Energy" is judged as "strong", it is quantified in " $h p=5$ ". The reciprocal comparison "Energy v/s Technical" results in " $h p=1 / 5$ ". An important advantage of the "AHP(blocks)" approach allows to model flexibly the values $\operatorname{HPM}_{(b} x$ b) with less computational efforts, less subjectivity, and better consistency in defining the hierarchy preferences. The block weights $(b w)$ are calculated as:

$$
\begin{aligned}
& \boldsymbol{b} \boldsymbol{w}_{\boldsymbol{e}}=\left(\prod_{\boldsymbol{e}=\mathbf{1}}^{b} \boldsymbol{h} \boldsymbol{p}_{e e}\right)^{\mathbf{1} / \boldsymbol{b}} /\left(\sum_{e=1}^{b}\left(\prod_{e=1}^{b} \boldsymbol{h} \boldsymbol{p}_{e e}\right)^{\mathbf{1} / \boldsymbol{b}}\right), \text { where } \boldsymbol{e}=\mathbf{1}, \ldots, \boldsymbol{b} . \\
& \sum_{e=1}^{b} b w_{e}=1
\end{aligned}
$$

For better results and simplified presentation a simulation of 3 variants of $H P M$ with different user's preference are proposed in Table 4.

Table 4. AHP Hierarchy Preference Matrices (HPM) for block weights definition

\begin{tabular}{|c|ccc|c|ccc|c|ccc|c|}
\hline Preferences & \multicolumn{3}{|c|}{ Balanced preferences } & \multicolumn{3}{|c|}{ Ecology-driven preference } & \multicolumn{4}{c|}{ Cost-driven preferences } \\
\hline Blocks & B1 & B2 & B3 & weight & B 1 & B2 & B3 & weight & B1 & B2 & B3 & weight \\
\hline B1:Technical & 1 & $1 / 2$ & $1 / 2$ & $\mathbf{0 . 2}$ & 1 & $1 / 4$ & $1 / 2$ & $\mathbf{0 . 1 4 3}$ & 1 & $1 / 2$ & $1 / 4$ & $\mathbf{0 . 1 4 3}$ \\
B2: Ecology & 2 & 1 & 1 & $\mathbf{0 . 4}$ & 4 & 1 & 2 & $\mathbf{0 . 5 7 1}$ & 2 & 1 & 1 & $\mathbf{0 . 2 8 6}$ \\
B3: Costs & 2 & 1 & 1 & $\mathbf{0 . 4}$ & 2 & $1 / 2$ & 1 & $\mathbf{0 . 2 8 6}$ & 4 & 2 & 1 & $\mathbf{0 . 5 7 1}$ \\
\hline Consistency & \multicolumn{3}{|c|}{$\mathrm{CR}=\mathbf{0} \%$ (excellent) } & \multicolumn{3}{c|}{$\mathrm{CR}=\mathbf{0} \%$ (excellent) } & \multicolumn{4}{c|}{ CR=0\% (excellent) } \\
\hline
\end{tabular}

To define objectively the weights of criteria in the blocks with more than one criterion (Block 1) the Entropy transformation is calculated as described in Step 3 of Section 2.

The definition of weights for the variant "Balanced preferences" is presented in Table 5.

Table 5. Entropy weighting of criteria with balanced "Ecology-to-Costs $(1: 1)$ " preferences

\begin{tabular}{|c|c|c|c|c|c|cc|}
\hline AHP Blocks & \multicolumn{3}{|c|}{ Block 1 -Technical } & Block 2 - Ecology & \multicolumn{3}{c|}{ Block 3 - Costs } \\
\hline Block weights & \multicolumn{3}{|c|}{$\mathbf{0 . 2}$} & $\mathbf{0 . 4}$ & \multicolumn{3}{c|}{$\mathbf{0 . 4}$} \\
\hline Criteria & $\mathrm{P}$ & OH & UL & tCO2/y & IR & IP & O\&M C \\
\hline SEnorm $=\mathrm{e}_{\mathrm{j}}$ & 0,820 & 0,772 & 0,992 & 0,852 & 0,927 & 0,976 & 0,912 \\
rebw (Blocks) & 0,317 & 0,299 & 0,384 & 0,400 & 0,329 & 0,347 & 0,324 \\
recw (Criteria) & 0,063 & 0,060 & 0,077 & 0,400 & 0,132 & 0,139 & 0,130 \\
\hline$d=1-e$ & 0,180 & 0,228 & 0,008 & 0,400 & 0,073 & 0,024 & 0,088 \\
debw (Blocks) & 0,434 & 0,548 & 0,018 & 0,400 & 0,394 & 0,130 & 0,476 \\
decw(Criteria) & 0,087 & 0,110 & 0,004 & 0,400 & 0,158 & 0,052 & 0,190 \\
\hline
\end{tabular}

The definition of weights for the "Ecology-driven preferences" is presented in Table 6.

Table 6. Entropy weighting of criteria with Ecology-driven preferences /Ecology-to-Costs (2:1)/

\begin{tabular}{|c|c|c|c|c|c|cc|}
\hline AHP Blocks & \multicolumn{3}{|c|}{ Block 1 - Technical } & \multicolumn{3}{c|}{ Block 2 - Ecology } & \multicolumn{3}{c|}{ Block 3 - Costs } \\
\hline Block weights & \multicolumn{3}{|c|}{$\mathbf{0 . 1 4 3}$} & $\mathbf{0 . 5 7 1}$ & \multicolumn{3}{|c|}{$\mathbf{0 . 2 8 6}$} \\
\hline Criteria & $\mathrm{P}$ & OH & UL & tCO2/y & IR & IP & O\&M C \\
\hline SEnorm $=\mathrm{e}_{\mathrm{j}}$ & 0,820 & 0,772 & 0,992 & 0,852 & 0,927 & 0,976 & 0,912 \\
rebw (Blocks) & 0,317 & 0,299 & 0,384 & 0,571 & 0,329 & 0,347 & 0,324 \\
recw (Criteria) & 0,045 & 0,043 & 0,055 & 0,571 & 0,094 & 0,099 & 0,093 \\
\hline$d=1-e$ & 0,180 & 0,228 & 0,008 & 0,571 & 0,073 & 0,024 & 0,088 \\
debw (Blocks) & 0,434 & 0,548 & 0,018 & 0,571 & 0,394 & 0,130 & 0,476 \\
decw (Criteria) & 0,062 & 0,078 & 0,003 & 0,571 & 0,113 & 0,037 & 0,136 \\
\hline
\end{tabular}

The definition of weighs for the "Cost-driven preferences" is presented in Table 7. 
Table 7. Entropy weighting of criteria with Cost-driven preferences /Ecology-to-Costs (1:2)/

\begin{tabular}{|c|c|c|c|c|c|cc|}
\hline AHP Blocks & \multicolumn{3}{|c|}{ Block 1 - Technical } & Block 2 - Ecology & \multicolumn{3}{c|}{ Block 3 - Costs } \\
\hline Block weights & \multicolumn{3}{|c|}{$\mathbf{0 . 1 4 3}$} & $\mathbf{0 . 2 8 6}$ & \multicolumn{3}{c|}{$\mathbf{0 . 5 7 1}$} \\
\hline Criteria & $\mathrm{P}$ & $\mathrm{OH}$ & $\mathrm{UL}$ & tCO2/y & IR & IP & O\&M C \\
\hline SEnorm $=\mathrm{e}_{\mathrm{j}}$ & 0,820 & 0,772 & 0,992 & 0,852 & 0,927 & 0,976 & 0,912 \\
rebw (Blocks) & 0,317 & 0,299 & 0,384 & 0,286 & 0,329 & 0,347 & 0,324 \\
recw (Criteria) & 0,045 & 0,043 & 0,055 & 0,286 & 0,188 & 0,198 & 0,185 \\
\hline$d=1-e$ & 0,180 & 0,228 & 0,008 & 0,286 & 0,073 & 0,024 & 0,088 \\
debw (Blocks) & 0,434 & 0,548 & 0,018 & 0,286 & 0,394 & 0,130 & 0,476 \\
decw (Criteria) & 0,062 & 0,078 & 0,003 & 0,286 & 0,225 & 0,074 & 0,272 \\
\hline
\end{tabular}

In the hybrid "AHP-block \&Entropy", approach the "real entropy criteria weights (recw)" are logical and directly deducted from primary data to reflect the real distribution of information. In opposite, the artificial calculation of "differed entropy criteria weights $($ decw $)$ " creates important redistribution of information in Block 1 and Block 3 which contain more than one criterion. In Block 1 the importance of "Utilization Life (UL)" is reduced to a neglected level: in the Balanced policy from 0.077 to 0.004 (19.25 times); in the Ecology-driven policy from 0.055 to 0,003 (18,3 times); and in the Cost/Efficiency-driven policy - from 0.055 to 0,003 (18,3 times). The situation is very similar in Block 3: the importance of "Construction period" is reduced in "Balanced policy" from 0.139 to 0.052 (2,7 times); in the Ecology-driven policy from0.099 to 0.037 (2.7 times); and in the Cost/Efficiency-driven policy - also from 0.198 to 0,074 (2.7 times). Data treatments depend on the researcher's concept and responsibility, but in real conditions they should be undertaken with common sense since such "differed content" may have serious consequences for users.

\section{Results and Discussion}

The ranking of results for best alternatives with MOORA in the four variants of weighs for the 3 different selection policies is displayed in Table 8. The stand-alone entropy method "SE" is $100 \%$ data-driven and it cannot take into account the users' preferences. Logically however the perturbation of results between "recw" and "decw" is very important - except for $\mathrm{t} \# 1$, \#2 and \#4 ranks all other results are different.

Table 8. Final ranking of MOORA assessment results for selection of RE projects

\begin{tabular}{|c|c|c|c|c|c|c|c|c|}
\hline \multirow{3}{*}{$\begin{array}{c}\text { Approach } \\
\text { Weights } \\
\text { Preference }\end{array}$} & \multicolumn{6}{|c|}{ Hybrid AHP Block \& Shannon Entropy } & \multicolumn{2}{|c|}{ Stand-alone Shannon Entropy } \\
\hline & \multicolumn{3}{|c|}{ recw } & \multicolumn{3}{|c|}{ decw } & recw & decw \\
\hline & $\mathrm{B}$ & $\mathrm{E}$ & $\mathrm{C}$ & $\mathrm{B}$ & $\mathrm{E}$ & $\mathrm{C}$ & Single variant & Single variant \\
\hline WA1 & 6 & 6 & 5 & 6 & 12 & 5 & 6 & 12 \\
\hline WA2 & 4 & 4 & 4 & 4 & 10 & 4 & $\underline{3}$ & 10 \\
\hline WA3 & $\underline{1}$ & $\underline{\mathbf{1}}$ & $\underline{\mathbf{1}}$ & $\underline{\mathbf{1}}$ & $\underline{\mathbf{2}}$ & $\underline{\mathbf{1}}$ & $\underline{\underline{2}}$ & $\underline{2}$ \\
\hline HA4 & 12 & 12 & 11 & 12 & 13 & 9 & 11 & 13 \\
\hline HA5 & 11 & 11 & 7 & 10 & 11 & 8 & 7 & 11 \\
\hline HA6 & 10 & 10 & 8 & 7 & 8 & 6 & 5 & 8 \\
\hline SA7 & 13 & 13 & 13 & 13 & $\underline{3}$ & 13 & 13 & $\underline{3}$ \\
\hline BA8 & 9 & 9 & 12 & 11 & 7 & 12 & 12 & 7 \\
\hline BA9 & 7 & 7 & 9 & 8 & 5 & 10 & 9 & 5 \\
\hline BA10 & 7 & 7 & 9 & 8 & 5 & 10 & 9 & 5 \\
\hline BA11 & 5 & 5 & 6 & 5 & 4 & 7 & 4 & 4 \\
\hline BA12 & $\underline{2}$ & $\underline{\mathbf{2}}$ & $\underline{2}$ & $\underline{\mathbf{2}}$ & $\underline{1}$ & $\underline{2}$ & $\underline{1}$ & $\underline{\mathbf{l}}$ \\
\hline BA13 & 3 & 3 & 3 & 3 & 9 & 3 & $\overline{8}$ & $\overline{9}$ \\
\hline
\end{tabular}

Legend: B - Balanced policy (Ecology/Costs); E - Ecology-driven policy; C - Cost-driven policy

In contrast, thanks to the block systematization in the combined "AHP-block \& Entropy" approach the policy preferences can be taken into account flexibly and correctly. Although 
values of AHP(blocks) weights were adjusted for modelling different preferences, the selections in the 3 variants of preferences in real entropy criteria weights ( $r e c w$ ) are very similar. In this case, this was predetermined by the empirical information contained in the original data-set. The top 4 alternatives with " $r e c w$ " for all 3 preference have the same ranking and are based on the more mature wind and biomass technologies (WA3>BA12>BA13>WA2).

The final assessments with the combined "AHP(blocks) \& Entropy" and MOORA approaches in our study are in accordance with the basic findings in the study of J.R. San Cristobal [17] received initially on the same data-set with the application of the classical AHP and VIKOR methods.

One of the purposes of our studies is to explore the repercussions of the "differed entropy" generated in eq. (8-9) on the robustness of MCDM process. The advantages of the combined "AHP(blocks) \& Entropy" method are confirmed by simulating selections in three different variants of preferences and assessments with the MOORA technique. The systematization of criteria in blocks allocates defined "quota" of importance to each block and this is space in which the "differed entropy" is limited redistribute the weights of criteria. If needed, additional AHP(blocks) decompositions can be performed for creating sub-blocks in selections with more criteria. This is often the case in MCDM for complicated investment projects with numerous technical, financial, social, and other criteria. A major advantage of such an approach is that at the sub-block levels the participation of experts can be very well structured to improve the consistency of their opinions. In addition, the DMs' involvement becomes more feasible and effective. At the same time, the application of artificially calculated weights risk to completely disregard the real needs of users and to increase uncertainty. For this reason, the data-driven methods based on a logical systematization should be preferred to the substitution of empiric facts by "differed reality".

\section{Conclusion and further research}

The methodological improvements and experiments in this study confirm that the enlargement of the traditional "Entropy" approach with the "block weighting" of criteria in AHP(blocks) provides an additional logical and accessible approach for multi-criteria analysis and effective support for the decision-making process. The comparative analysis of the variants with real and differed weights of criteria confirm our preferences for direct measuring of diversity based on real data and sets a basis for further research in other areas and evaluation techniques used in MCDM.

From a practical point of view, this study provides a useful basic framework for analyzing RE investment projects and improving the MCDM process with approaches tailored to preferences and budgets of different users, regions, and technologies under conditions of pandemic and economic restraints. Another result is the confirmation that benefits from economies of scale are well expressed in the all types of technologies based on different RE sources. This is important for convincing the users to be orientated for more performant equipment if they can be realized in their region, and at the same time to have a flexible and portfolio-oriented policy. The combination of projects based on different technologies will be critical for building diversified and reliable energy-generating systems. The data set employed in this system includes alternatives that were ranked without controversies in the preference profiles. In practice, the real situation is expected to become more uncertain with the progress in the different technologies and the increase of competition between manufacturers of different systems and equipment, which would require considering larger numbers of criteria and alternatives with increased number of experts.

Further applied research should include the application of "AHP(blocks) \& Entropy"approach with different final evaluation techniques, such as TOPSIS, VIKOR, WPN, 
ELECTRE, and others which will provide integrated and more reliable methods for selecting investment projects. Taking into account that the migration to "zero carbon emissions" energy model can be achieved during a reasonable transition period of 20-30 years, the future research should include other technologies (hydrogen, gas, coal, nuclear an hybrid projects) depending on the specifics of different countries and geographical regions.

This research is supported by the Bulgarian FNI fund through the project "Modeling and Research of Intelligent Educational Systems and Sensor Networks (ISOSeM)", contract P-06-H47/4 from 26.11.2020.

\section{References}

1. Brauers, W., Optimization methods for a stakeholder society (Boston: Kluwer Academic, 2004)

2. Barzilai, J., Springer. Int. Ser. in Oper. Res. \& Man. Sci. 142, pp. 57-86 (2010)

3. Saaty, T., Principia Mathematica Decernendi: Mathematical Principles of Decision Making (Pittsburgh, Pennsyl.: RWS Publ., 2010)

4. Zavadskas E., Turskis Z., Tech. and Econ. Devel. of Econ. 17(2) pp.397-427 (2011).

5. Genova, K., Kirilov, K., Guliashki, V., Cyber. and Inf. Tech., 2, pp. 3-22 (2015)

6. Petrov, P., Kostadinov, G. and al., Int. Conf. Automat. and Inform. (ICAI), Varna, Bulgaria, pp. 1-5, (2020) doi: 10.1109/ICAI50593.2020.9311351

7. Opricovic, S., Tzeng, G.H., Eur. J. Oper. Res.,156, pp. $445-55$ (2004)

8. Hwang, C.L., Yoon, K. Multi-attribute decision making: methods and applications. Berlin: Springer-Verlag; (1981)

9. Georgopoulou, E., Lalas, D., Papagiannakis, L., Eur. J. Oper. Res. 103(1), pp. 38-54 (1997)

10. Golabi, K., Kirkwood, C.W., Sicherman, A., Manag. Sci., 22(2), pp. 174-89, (1981)

11. Lee, A.H.I., Chen, H.H., Kang, H.Y., Renew. Ener. J., 34, (2009).

12. Ilchev, S., Petkov, D., Andreev, R., Ilcheva, Z., J. Cyb. and Inf. Tech., 19 (3), pp. $137-$ 153, (2019)

13. Alexandrov, A., Andreev, R., Ilchev, S., Batchvarov, D., Boneva, A., Ivanov, S., Doshev, J., in HPC 2019 Conf., Stud. in Comp. Intel. 902, (2020)

14. Brauers, T., Zavadskas, E., Tech. and Econ. Devel. of Econ., 15(2), pp.352-375 (2009)

15. Anysz, H., Nica, A., and al., J. Symmetry, 1/46, (2021),

16. Attri, R., Grover, S., Int. J. of Sys. Ass. Eng. and Man., 5(3), pp. 320-328 (2014)

17. San Christobal, J.R., Renew. Energ. J. 36, pp.498-502 (2011)

18. Shannon, C., Bell Sys. Tech. J. 27(3), pp.379-423 (1948)

19. Saaty, T., Peniwati, K., Group Decision Making: Drawing out and Reconciling Differences (Pittsburgh, Pennsyl. RWS Publ., 2008)

20. Borissova, D., Comptes rend. de l'Acad. Bulg. des Scien., 71(11), pp. 1520-1527 (2018)

21. Korsemov, D., Borissova, D., Adv. Model. and Optim. J., 20(1), pp. 101-112 (2018), ISSN: $1841-4311$

22. Saaty, S., Decision Making for Leaders: The Analytic Hierarchy Process for Decisions in a Complex World (Pittsburgh, Pennsyl. RWS Publ., 2008) ISBN 978-0-9620317-8-6

23. Forman, E., Saul, G., Oper. Res. J., 49 (4), pp. 469-487 (2001) doi: 10.1287/opre.49.4.469.11231

24. Petrov. I., in Conf. "TechSys 2021", 27-29 May 2021, Plovdiv, Bulgaria. 8 pages, in print., http://techsys.tu-plovdiv.bg/index.html\#HOME 